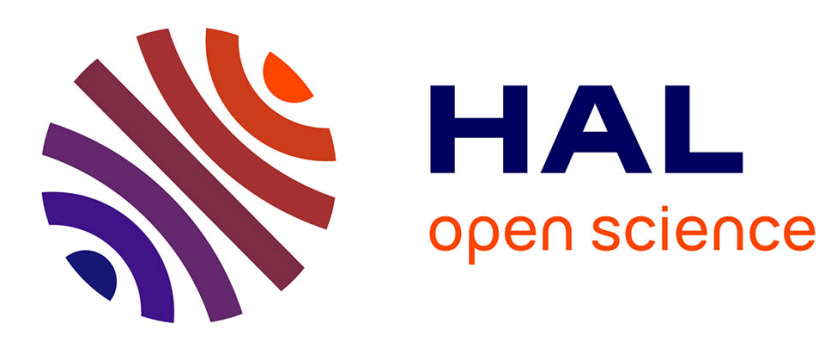

\title{
Les bébés face aux supports numériques
}

Laurence Berdot-Talmier

\section{To cite this version:}

Laurence Berdot-Talmier. Les bébés face aux supports numériques. Spirale, 2017, Les bébés et les écrans, 3 (83), pp.56-61. 10.3917/spi.083.0056 . hal-02150213

\section{HAL Id: hal-02150213 \\ https://hal-univ-tlse2.archives-ouvertes.fr/hal-02150213}

Submitted on 7 Jun 2019

HAL is a multi-disciplinary open access archive for the deposit and dissemination of scientific research documents, whether they are published or not. The documents may come from teaching and research institutions in France or abroad, or from public or private research centers.
L'archive ouverte pluridisciplinaire HAL, est destinée au dépôt et à la diffusion de documents scientifiques de niveau recherche, publiés ou non, émanant des établissements d'enseignement et de recherche français ou étrangers, des laboratoires publics ou privés. 


\title{
Les bébés face aux supports numériques
}

\author{
Laurence Berdot-Talmier
}

Psychologue

Chargée d'enseignements en psychologie, UT2J

Doctorante en psychologie du développement, UMR LISST-CERS (UMR 5193), UT2J

\section{Résumé :}

La Technologie numérique étant omniprésente dans la vie des bébés, cet article retrace l'impact des médias et tout particulièrement celui de la télévision sur le développement des tout-petits. Bien que la plupart des experts soulignent l'impact négatif de ce média chez les bébés, certains aspects positifs méritent d'être examinés. En outre, les technologies mobiles et tactiles, en représentant une expérience interactive se doivent d'être considérées différemment.

Mots-clés : bébés- télévision- technologie tactile-exposition-développement

\begin{abstract}
Babies facing the media

As digital technology is prevalent in the life of infants, this paper discerns the impact of media and more specific the impact of television on the development of the baby. Even though many studies point out the negative impact of the television on babies, certain positive aspects have to be further examined. Additionally, the tactile and mobile technologies represent an interactive experience which has to be considered in another point of view.
\end{abstract}

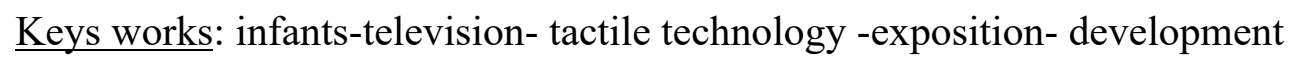




\section{Introduction}

A l'heure actuelle, les nourrissons et les tout-petits vivent dans un environnement empli de médias de toutes sortes. Depuis le début de leur vie, ils grandissent généralement en présence des Technologies Numériques de l'Information et de la Communication (TNIC) ou plus couramment en face des écrans ${ }^{1}$. Malgré la montée en puissance des «nouveaux » médias (téléphones portables, tablettes tactiles...), la télévision et les vidéos restent les expériences médiatiques les plus courantes pour les plus jeunes enfants. Il n'y a donc rien de surprenant à ce que les nourrissons soient exposés, de façon intentionnelle ou non, à une quantité importante de contenus médiatiques.

\section{A quel âge devrait-on laisser les tout-petits devant les écrans ?}

Que ce soit en France ou aux Etats-Unis, des recommandations ont vu le jour afin de sensibiliser les parents à cette exposition en fonction de l'âge des enfants. En France, suite aux travaux du groupe d'expert en 2008 sur l'impact des chaînes télévisées sur le tout-petit enfant, l'avis de la Direction Générale de la Santé a émis des préconisations en ce qui concerne les limites temporelles que devraient fixer les parents. Tisseron ${ }^{2}$ préconise la règle des «3-6-9-12», avançant l'idée que les écrans sont à proscrire avant l'âge de 3 ans. L'académie américaine de pédiatrie ${ }^{3}$, en se centrant sur les périodes développementales distinguées en psychologie cognitive, mentionne que cette exposition est à bannir pour les bébés de moins de 2 ans.

\section{Exposition des bébés à la télévision}

« Avant même de marcher, le bébé regarde la télévision, blotti dans les bras de sa mère. Il est attiré par cette boîte de lumière et de sons et, dès qu'il sait ramper, il se déplace vers elle $»^{4}$.

Un sondage nationalement représentatif auprès de parents d'enfants âgés de 0 à 8 ans aux Etats-Unis ${ }^{5}$ a été mené afin d'analyser les différents modes d'utilisation des médias chez les jeunes enfants. Cette enquête a révélé que les bébés de moins de 2 ans ont une exposition

\footnotetext{
${ }^{1}$ Huerre \& Vlachopoulou, 2015

2 Tisseon, 2009

${ }^{3}$ American Academy of Pediatrics, 2011

${ }^{4}$ Bourcier, 2010, p.19

${ }^{5}$ Rideout, 2013
} 
quotidienne moyenne de 58 minutes aux médias, et que les tout-petits de 2-4 ans y ont une exposition moyenne de 1 h58 minutes. Bien que la télévision soit le media le plus usité par les tout-petits, d'autant plus que 16\% des bébés de moins de 2 ans disposent d'un téléviseur dans leur chambre et qu'ils sont $37 \%$ à 2-4ans, 38\% des bébés de moins de 2 ans utilisent les écrans mobiles et tactiles et ce pourcentage passe à $80 \%$ chez les enfants âgés de 2 à 4 ans. De plus, tout type de médias confondus, pour les tout-petits, cette exposition est plus importante lorsque les mères ont un faible niveau d'éducation et dans les familles avec un faible statut socio-économique.

Beaucoup d'enfants, même très jeunes sont si familiers des médias qu'ils sont capables d'allumer eux-mêmes la télévision (38\% des enfants âgés de 6 à 23 mois et $82 \%$ entre 2 et 3 ans), de changer de chaînes avec la télécommande ( $40 \%$ à 6-23 mois et 54\% à 2-3 ans) et même de positionner un DVD dans le lecteur afin de le visionner (7\% à 6-23 mois et $42 \%$ à 23 ans). Cette familiarité leur procure un certain degré d'autonomie par rapport aux médias ${ }^{6}$.

Dans de nombreux foyers avec enfants, la télévision est pour la plupart du temps, voire tout le temps allumée, que quelqu'un la regarde ou non. Il faut donc également prendre en compte cette exposition de « fond » que le tout jeune enfant pourra voir et/ou entendre même s’il ne la regarde pas activement. Une étude récente a fait état d'une exposition globale à la télévision d'une durée moyenne de 5 heures et demi par jour chez les bébés âgés de 8 à 24 mois 7 .

Depuis des millénaires, les individus ont été influencés par les médias qu'ils utilisent et consomment, et bien souvent, les effets engendrés sont globalement perçus comme négatifs pour et sur les personnes. D'une façon générale, la technologie a toujours suscité de l'inquiétude, et tout particulièrement en ce qui concerne son adoption par les plus jeunes ${ }^{8}$. Aussi, avant même de s'intéresser aux effets positifs pour ces très jeunes enfants, compte tenu du nombre de médias à disposition, le débat, en partie influencé par la «panique morale» tend à considérer l'impact négatif de cette technologie pour les bébés.

\section{Pourquoi de telles inquiétudes ?}

La réponse implique les transformations remarquables qui vont se produire au niveau du développement cérébral durant les 3 premières années de vie. Du fait de la plasticité cérébrale, des modifications vont se produire, en réponse directe aux stimulations externes,

\footnotetext{
${ }^{6}$ Rideout \& Hamel, 2006

${ }^{7}$ Lapierre, Piotrowski \& Linebarger, 2012

${ }^{8}$ Berdot-Talmier \& Zaouche Gaudron, 2017
} 
faisant des premières expériences de vie, des éléments essentiels aux trajectoires développementales 9 .

Durant les 6 premiers mois de vie, le bébé va être captivé par les couleurs, le flux d'images bidimensionnelles et de sons provenant de la télévision, mais il n'a pas encore la capacité de traiter correctement ce qu'il regarde ou de maintenir son attention sur une longue période. Plus tard, au cours de la $1^{\text {er }}$ année et au-delà, au regard du développement cognitif et perceptif, le tout-petit sera en mesure de reconnaître les personnages et les objets sur l'écran. Bien qu'à la naissance, le cerveau des tout-petits ne soit pas « câblé » pour comprendre ce qu'il voit sur les écrans, il est néanmoins bien équipé pour comprendre les interactions sociales.

\section{Comment les écrans affectent-ils les tout-petits ?}

Les experts ${ }^{10} \mathrm{~s}^{\prime}$ accordent pour signaler que les écrans vont impacter le développement des tout-petits par divers aspects.

La présence de la télévision va entrainer une réduction tant qualitative que quantitative dans les interactions parent-bébé. Ce déclin dans les interactions se conçoit à la fois en terme de diminution dans les communications et les échanges ainsi que dans un moindre engagement des parents auprès de leurs bébés ce qui pourra avoir des répercussions sur le développement de l'enfant. Ce constat appuie l'hypothèse selon laquelle la télévision peut distraire les parents ou interférer sur leurs capacités de répondre de façon adéquate au bébé.

La présence de la télévision va également venir affecter les activités de jeux du tout-petit pourtant essentielles à son développement. En effet, le jeu, le dessin, le coloriage et l'exploration sont des activités qui permettent aux bébés et aux tout-petits d'aiguiser leurs capacités cognitives, socio-émotionnelles et leurs habiletés motrices, mais les distractions fréquentes, causées par la télévision en arrière fond, peuvent entraver ces processus. Avec une télévision allumée, les jeunes enfants seront moins en capacité de se concentrer sur le jeu actif. Cette exposition aux écrans va également avoir un impact sur les parents, alors même que le jeu leur offre la possibilité de s'engager pleinement dans l'activité avec l'enfant, que ce soit en termes de participation active ou par l'accompagnement verbal. Selon Lurçat ${ }^{11}$ «l'association du langage et des activités de l'enfant est nécessaire, non seulement pour son développement harmonieux, mais également pour l'acquisition du langage descriptif, car le

\footnotetext{
${ }^{9}$ Bach, Houdé, Léna \& Tisseron, 2013

${ }^{10}$ American Academy of Pediatrics, 2011

11 in Bourcier, 2010, p.98
} 
langage fait corps avec l'activité, il en est un aspect important». Ceci est d'autant plus préjudiciable que la croissance du vocabulaire des tout-petits est directement liée au temps d'interactions verbales que les parents vont partager avec le bébé.

Cette exposition à la télévision va également avoir un impact négatif sur le développement cognitif et langagier du tout petit. Une étude ${ }^{12}$ a pu mettre en évidence que les bébés exposés à 2 heures ou plus de télévision par jour avant l'âge de 12 mois étaient six fois plus susceptibles de développer un retard de langage comparativement aux tout-petits qui ont commencé à regarder la télévision après leur $1^{\text {er }}$ année. De plus, le développement linguistique des tout-petits peut être affecté du fait qu'ils vont passer plus de temps à écouter la télévision, ce qui est un langage réceptif, plutôt que de s'engager activement dans un langage productif dirigé vers autrui.

Au-delà de ces aspects négatifs, il est aussi très important de reconnaître que la télévision peut avoir un impact positif dans la vie des tout-petits. Bien que les bébés ne perçoivent pas ce média de la même façon que les adultes, petit à petit, avec le développement cognitif il vont progressivement accroître leurs habiletés télévisuelles. Nous avons vu que bien que la compréhension de la télévision est minimale chez le nourrisson et que son attention peut être purement réactive, à mesure qu'il grandit, la visualisation de programmes éducatifs ou récréatifs qui leur sont spécifiquement dédiés peuvent être compréhensibles et refléter un traitement cognitif plus mâture. Ces programmes vont encourager une participation active des tout-petits, avec des personnages qui vont directement s'adresser à eux et où les jeunes enfants vont alors pouvoir chanter, danser, imiter les comportements du personnage, pointer des objets, le tout avec enthousiasme ${ }^{13}$. Les parents confirment que les enfants sont activement impliqués et que le développement linguistique est, par certains aspects, directement en lien avec la télévision ${ }^{14}$. Les enfants d'âge préscolaire vont être en capacité d'utiliser des mots, d'écouter des histoires ou des chansons en s'impliquant activement dans le refrain par exemple, d'écouter et d'utiliser le langage parlé, de maintenir une écoute attentive et réactive, tout comme ils vont au fur et à mesure de leur construction être en capacité de comprendre les valeurs et les codes de conduites socialement attendues. Le temps passé devant la télévision peut également être un moment de partage et d'échange au sein de la cellule familiale. Des programmes diversifiés et adaptés à l'âge des enfants, tels que des

\footnotetext{
${ }^{12}$ Chonchaiya \& Pruksananonda, 2008

${ }^{13}$ Lemish, 2007

${ }^{14}$ Marsh, Brooks, Hugues, Ritchie, Roberts \& Wright, 2005
} 
documentaires ou des programmes culturels, peuvent les aider à se créer une image du monde, à découvrir le milieu de la musique et de l'art etc.

Finalement, de par le partage d'activités, d'échanges verbal et non verbal, les parents vont fournir aux tout-petits l'accès aux représentations qui leur permettront de comprendre et d'utiliser les systèmes qui représentent la réalité, y compris les représentations de la réalité audiovisuelles.

\section{Les écrans tactiles}

Etant donné les caractéristiques uniques des technologies mobiles, c'est-à-dire, leur portabilité et leur interactivité, il est important de se questionner sur l'impact de ces technologies sur le développement du tout-petit. En effet, pour l'instant, avec le manque de données sur ce sujet, nous pouvons supposer que l'utilisation de ces technologies comme moyen passif de visionner des vidéos montrera des effets similaires chez les tout-petits enfants en développement. Bien souvent, en raison de cette portabilité, les parents utilisent les médias mobiles et tactiles comme outil de régulation comportementale afin de maintenir les toutpetits à la fois apaisés et occupés durant les activités et routines quotidiennes ${ }^{15}$. Bien que ces dispositifs mobiles puissent être utiles à court terme pour occuper les enfants, une sur exploitation de ces outils à cette fin pourrait être préjudiciable sur le développement socioémotionnel ultérieur.

« il n'est pas question de faire de ces supports des baby-sitters, au risque d'enfermer le petit enfant dans un mode de relation d'objet problématique ${ }^{16}$

Toutefois, les médias mobiles et tactiles diffèrent considérablement de la télévision. En effet, contrairement à la télévision où le jeune enfant pourrait, par moment, être passif ou être considéré comme tel, et donc exposé au contenu, les technologies mobiles vont lui permettre d'être en interaction avec l'outil. De par cette fonction tactile, ils éliminent la nécessité d'avoir des périphériques externes, tels que clavier ou souris, qui nécessitent un certain niveau de savoir-faire. De nombreuses applications sont disponibles pour les jeunes enfants avec une conception intuitive qui peuvent être propices pour le développement sensori-moteur des toutpetits ${ }^{17}$. Les bébés développent leur connaissance perceptuelle du monde qui les entoure en interagissant avec les autres, les jouets et les différents objets disponibles dans leur

\footnotetext{
15 Tisseron, 2009

${ }^{16}$ Huerre \& Vlachopoulou, 2015, p. 16

${ }^{17}$ Bach, Houdé, Léna \& Tisseron, 2013
} 
environnement. Avec la maturation et au travers des multiples modalités sensorielles, ils vont petit à petit coordonner les informations reçues, il est donc crucial que des recherches se développent afin de savoir quels seront les impacts des interactions régulières avec ces interfaces au cours de cette phase développementale particulièrement critique pour le développement.

\section{Conclusion}

L'utilisation de la télévision semble associée, pour certains, à un déplacement d'activités et d'interactions essentielles pour un développement harmonieux du tout-petit, alors que pour d'autres, elle semble être une source d'apprentissage et de partage. Concernant l'interactivité des technologies tactiles, de par le manque de données à ce sujet, nous pourrions nous attendre à ce que cette utilisation rencontre les mêmes controverses au niveau scientifique. Toutefois, il semble que l'utilisation des médias interactifs puisse être judicieuse pour les enfants de moins de 2 ans, dont l'intelligence est, à cet âge-là, avant tout « sensori-motrice ».

Les parents ont un rôle important dans la qualité et la quantité d'expériences que les enfants vont expérimenter sur ces outils.

L'accompagnement par les adultes est fondamental pour guider le jeune enfant dans ses activités numériques ludiques, afin qu'il soit en mesure de mettre du sens au monde qui l'entoure $^{18}$. L'écran tactile, dans un contexte relationnel, pourrait donc être considéré comme un objet permettant au jeune enfant, au même titre que les autres objets à dispositions, d'explorer et de développer ses connaissances.

\section{Bibliographie}

AMERICAN ACADEMY OF PEDIATRICS. 2011. « Media use by children younger than 2 years », Pediatrics, n $^{\circ} 128$, p. 1040-1045.

BACH, J.F., HOUDE, O., LENA, P., \& TISSERON, S. 2013. L'enfant et les écrans. Un avis de l'Académie des Sciences, Paris, Le Pommier.

BERDOT-TALMIER, L., \& ZAOUCHE GAUDRON, C. 2017 (à paraître). «Les Technologies Numériques de l'Information et de la Communication dans l'espace familial » dans A. VINAY (sous la direction de), Psychologie de la famille aux différentes étapes de la vie : approche clinique et développementale, Paris, Dunod.

${ }^{18}$ Huerre \& Vlachopoulou, 2015 
BOURCIER, S. 2010. L'enfant et les écrans, Montréal, Editions du CHU Sainte-Justine.

CHONCHAIYA, W., \& PRUKSANANONDA, C. 2008. «Television viewing associates with delayed language development », Acta Paediatrica, n 97, p. 977-982.

HUERRE, P., \& VLACHOPOULOU, X. 2015. «Grandir à l'heure du numérique », Soins Pédiatrie Puériculture, $\mathrm{n}^{\circ} 282$, p. 14-20.

LAPIERRE, M. A., PiOTROWSKI, J. T., \& LINEBARGER, D. L. 2012. «Background television in the homes of US children », Pediatrics, $n^{\circ} 130$, p. 839-846.

LEMISH, D. 2007. Children and television: A global perspective, Oxford, Blackwell.

MARSH, J., BROOKS, G., HUGHES, J., RITCHIE, L., ROBERTS, S., \& WRIGHT, K. 2005. Digital beginnings: Young children's use of popular culture, media and new technologies, Literacy Research Centre, University of Sheffield

TISSERON, S. 2009. Les dangers de la télé pour les bébés, Toulouse, Eres.

RIDEOUT, V. 2013. Zero to eight: Children's media use in America in 2013, San Francisco, CA, Common Sense Media.

RIDEOUT V, HAMEL E. 2006. The Media Family: Electronic Media in the Lives of Infants, Toddlers, Preschoolers and Their Parents, Menlo Park, CA, Kaiser Family Foundation. 